\title{
User Interface Design as a Catalyst for Organisation Change
}

\author{
Professor Mark Anderson \\ Centre for Data Analysis and Representation \\ Department of Computing, Edge Hill University \\ Ormskirk, UK \\ mark.anderson@edgehill.ac.uk
}

\author{
Max Gilbert \\ Department of Computing \\ Edge Hill University \\ Ormskirk, UK \\ gilbertm@edgehill.ac.uk
}

\begin{abstract}
This paper presents a case study that explores the impact of a re-engineering project on a Small to Medium Enterprise in the United Kingdom as a means of delivering improved employee performance. In this paper, the link between the actions undertaken my teams of co-workers and the relationship with the environment provided by IT systems is considered. As an organization evolves over an extended period of time, both the IT systems and also the working practices employed to complete the day to day tasks essential to meet the needs of an organisation's customers, particularly in the service sector, rapidly become unfit for purpose. This soon becomes an issue both in meeting the needs of customers and also in meeting regulatory requirements. The case study considered in this paper reviews the requisite practices adopted by a service provider in the wetstock management industry, and the positive impact that the company realized through the re-engineering of both IT systems and business workflows. The central driver for the changes implemented was a need identified in the business to implement improved visualization of data relating to working practices in the organization and its clients. Through a regular review process, issues were identified in a key area of the business that impacted upon the time taken to complete essential tasks. The underlying reasons for this inefficiency were analysed and, through the Agile software engineering methodology, a solution was designed to resolve these issues.
\end{abstract}

User Interface design; business workflow re-engineering; teamworking practices; visualizing management information.

\section{INTRODUCTION}

The performance, and success, of any business in the service industries is largely driven by the quality of the provision that the business offers to its customers. Indeed, the quality of service becomes a key factor for those businesses that qualify their levels of provision through Service Level Agreements (SLAs) that exist between the business and its clients. Whilst quality assurance may be a key driver to facilitate a point of understanding between a business and its clients, the importance of presenting quality assurance methods and metrics rises in industry sectors where regulatory requirements impose operational conditions upon a service provider. Indeed, this is a situation that becomes more complex as businesses expand to provide services for clients around the world as regulatory requirements often change from country to country. Often these changes to regulations are significant. An added complexity may arise when the service is provided to clients that deal in high-value or high-risk products.

The business that is the focus of this case study operates within such a field; that of wetstock management. The clients of the business deal in high value commodities that require close monitoring of the storage and retail environment. This is due to the value of the goods and also the chemical constituents of the products. Within this sector, monitoring of the storage environment for the commodities is critical as environmental changes may indicate contamination, theft or other loss of the goods. The business is a Small to Medium Enterprise (SME) in the United Kingdom and has a client base that consists of a number of high profile multi-national organisations. It is therefore critical for the business to react quickly to changing demands within the market in which they trade, and to provide secure and timely outcomes for their clients. Through active engagement with their client base, the business has developed new technologies and working practices to support the operation of their clients.

Whilst the delivery of the service is important to clients at a holistic level, the business must determine means through which they can not only meet but improve upon, and indeed exceed, the standards that are set for their clients. In order to achieve this goal, the business must ensure that its own underpinning workflows are optimized for enhanced performance of the key operations. One approach to achieve this might be considered the implementation of IT support systems to complement the working patterns of the business employees. However, it is often the higher level workflows that can be reviewed to implement the greater impact.

The addition of the new technologies and service levels led to a more competitive organisation and led to a significant period of growth, up $100 \%$ within a 2 year period. To complement the growth in the business, a large number of new staff have been employed. This led to an inevitable demand for in-house training to prepare new staff members for the analysis roles that would be assigned. However, it soon became clear that the business faced key challenges in meeting the demands of their rapid growth. A key observation was that the management teams within the business did not have access to the data they required, or that the data was not readily available. Whilst the data could be extracted from the existing processes, the length of time this 
took was significant. Below the management level the individual employees also encountered an increasing number of tasks, more complex tasks and an increase in difficulty to access information. Adding an additional layer of complexity, it was found that the new members of staff faced increasingly intricate procedures in training which led to lengthened training programmes and increased confusion amongst staff.

Whilst the business was able to maintain their role as market leaders within their business sector, the need for change became a key driver for the Quality Department to evaluate existing workflows and implement streamlined procedures. As many of the tasks were performed through a bespoke software system, the business opted to refactor both the software and the workflows through a single project. As the success of the project was crucial to retain a leading status in the sector, the business entered into a partnership with a research centre to embed a dynamic approach for the existing, and future, re-engineering processes. This paper reports upon the approach that was adopted and the impact that the redesigned workflows and software system had upon the business.

\section{EXISTING PROCESSES}

The service that the company provides to its clients focuses on the management of wetstock determining and understanding events that occur. These events are categorised and may be identified either via data feeds from sensors attached to wetstock storage devices, or through custom sensing devices that collect and transmit data feeds from client sites. As data is gathered from each site, analysts interpret the data to identify unusual occurrences that are deemed to be alerts. Each alert may be classified as an event that requires intervention, or as a temporary inaccuracy in the data. Accurate interpretation of this data is fundamental for the successful completion of analyst's weekly tasks.

The company utilized a bespoke data management system. Using this system, the company are able to collate data from their clients to identify unexpected conditions, deemed to be alerts. Teams of analysts, under the supervision of Consult-ants and Contract Managers, review the incoming alerts to determine the most appropriate action to be taken. It may be considered that there are two distinct areas of data interpretation that is embodied within the system: the analysts interpret the individual alerts or content of the system, whereas the Contract Managers are making holistic interpretations relating to the ongoing processes with which their teams are engaged [1]. Reporting of information from the system is integral within the operations department to ensure targets are met and for the company department to ensure the wider goals of providing evidence that the Quality Management System is maintained. To support this, the company designed workflows that determined the appropriate procedures to be followed.

Within the operations department the Analyst requires information to ensure their own targets are met, the Consultant to ensure their analysts have met their targets and the Contract Manager to ensure their Consultants were on track and to be able to provide the relevant information to the Directors. Within the core areas of analysis each report to be generated required a different manual report to be produced at the analyst level. This would often mean the combining of multiple ad hoc reports from different sections of the data management system. The output of such ad hoc reports must then be manually copied and pasted to form one holistic report for one of the core areas of the business. It may be deemed, therefore, that this function within the company is highly process-based, requiring the integration of outputs from a range of diverse business processes [2]. An additional level of complexity is that the system would not necessarily be able to run the same information at either a team or department level resulting in the combing of reports between the Analyst to the Director more complex.

The internal organisation of the business that was analysed for this project consisted of teams of analysts who would collate data from a bespoke software system. The role of the analyst is to evaluate alerts raised through the software. An alert is triggered when an unexpected or unexplained condition arises in the data transmitted from a client site. The teams are overseen by a Consultant in a supervisory capacity. The Consultants are supervised by Contract Managers who have direct responsibility to the clients. As each client may operate at a number of sites, teams are allocated to clients such that one team is responsible for the management of a client's data. Equally, the sites are allocated to individual analysts. An exception to this situation arises when analysts are allocated to weekend working. When this happened, the analyst would require access to the data from additional clients than they normally process. The original software system was designed in such a way that any analyst could effectively masquerade as another analyst to facilitate weekend working schedules.

One of the core activities that the Analysts, Consultants and Contract Managers must undertake is the generation of reports to track the number of alerts processed against the number of alerts in the system. Reporting of information from the system is integral within the operations department to ensure targets are met and for the company department to ensure the wider goals of providing evidence that the Quality Management System is maintained. As an Analyst reviews an alert, then a decision is made regarding the most appropriate action to be taken. For example, some alerts are deemed to be false if the trigger is derived from pre-defined 
environmental conditions. At the other extreme, some alerts require urgent intervention. As an Analyst processes an alert, then the status of the alert is changed to reflect the actions that have been taken. The significance of the report generation is therefore two-fold. It firstly offers a means by which the Consultants and Contract Managers, as senior members of analyst staff, can monitor the actions and the productivity of their respective teams. Secondly, the reporting tools offer a means through which training needs can be identified and actioned; for example, should a large number of alerts be incorrectly interpreted by an analyst then the Consultant can intervene and instigate additional training. This implies that a two-phase approach to the management of data within the system is supported [1].

The core areas of analysis have different timescales and targets from morning and daily to weekly and monthly. As a result, various reports would be required at different time intervals, which added to the interruption of actual operations tasks; while reports are being generated the system was placed under additional stress and directly affected workload continuity.

Whilst the generation of reports has become a core activity for the analyst teams within the company, the software supporting this business function had become increasingly cumbersome. As the workflow processes around the analysis and quality management tasks had evolved since the inception of the business, the software had undergone numerous changes and updates in an attempt to meet business needs. Within the core areas of analysis each report to be generated required a different manual report to be produced at the analyst level. This would often mean the combining of multiple ad-hoc reports from different sections of the data management system. The output of such ad-hoc reports then had to be manually copied and pasted to form one holistic report for one of the core areas of the business, demonstrating the process-based nature of the business [2]. An additional level of complexity is that the system would not necessarily be able to run the same information at either a team or department level resulting in the combing of reports between the Analyst to the Director more complex. As an additional complexity, the core areas of analysis have different timescales and targets from morning and daily to weekly and monthly. As a result, various reports would be required at different time intervals which added to the interruption of actual operations tasks; while reports are being generated the system was placed under additional stress and directly affected workload continuity.

To achieve each daily task, an analyst would need to perform a six-step process. The process is represented in Figure 1.

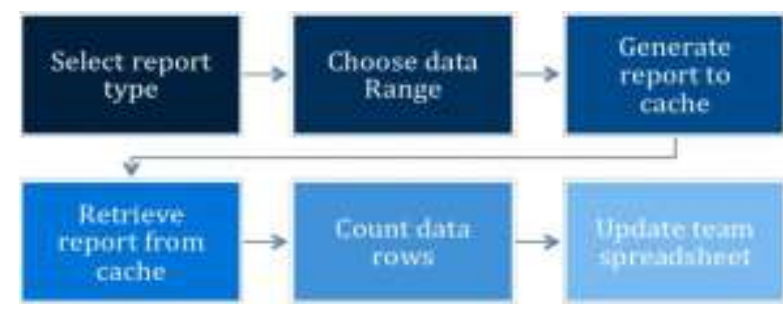

Figure 1: Stages of report generation

There are clearly a significant number of steps that the analysts must undertake in order to successfully generate a report. Timing the analysts actions, it was determined that the average time taken for each step was

- Step $1+2$ - 5 minutes

- Step 3 - 1 minute 45 seconds

- Step 4 - 45 seconds

- Step 5 - 5 minutes

The evaluation process initially considered a trial group of 80 analysts. For these users, completing the reports on a weekly basis would result in 16.6 hours of work per week. However, it was also determined that in order for the Contracts Managers to collated accurate data then the analysts would need to perform these tasks once per day. This would equate to 83 hours of work per week.

The difficulty faced by the analysts lay in the software system that they used to access and process the data. As the system had been developed over a 20-year period, the software had become difficult to operate. Specifically, the analysts and the Contract Managers had to navigate a number of screens in order to access and process the requisite data. Equally the presentation of the data was deemed to be inadequate, and required the analysts to spend extended periods of time understanding the data. Equally the Contract Managers were undertaking protracted steps to collate team performance reports. Ultimately, the outcome of each of these challenges related to the use of the software system led to two issues of concern to the business. Firstly there existed an opportunity that the generated reports were inaccurate; either through misinterpretation of the data or as a result of the protracted analysis processes leading to outdated data being analyzed. The second concern related to the potential for analysts to identify alternative means of completing their daily tasks and thereby bypassing the workflows that the company had designed. The latter issue was a major point of concern for the company as the service they provided directly impacted upon the Quality Management procedures of their clients. 
In redesigning a new software system with enhanced features and data presentation, the business set out to improve the workflows that underpinned the tasks that the teams completed. Whilst the outcomes of the workflows were fixed, being designed to meet both customer and regulatory requirements, the opportunity existed to enhance team working and management practices to facilitate improved quality management for clients. To achieve a successful outcome for the refactoring project, the company applied theory that supported virtual teamwork to integrate improved levels of technology-mediated communication within the teams, and between the Contract Managers and the senior management teams.

\section{TEAM WORKING}

A core issue that was identified early in the analysis of existing software tools was the restricted communication facilities that existed. The lack of tools to support cooperation between co-workers offered significant challenges as the teams grew in size and the Contract Managers became responsible for increasing numbers of teams. Whilst there was a level of independence that existed between analysts, as an analyst may perform a number of their daily tasks without assistance from others in the team, there were a number of operations in the business that correlated to the support requirements of virtual teams. Taking a definition of a virtual team as a team that "technology-mediated communication, while crossing the boundaries of geography, time, culture and organization to accomplish an interdependent task" [3]. For the expanding business, these issues were soon to become important, particularly as

1. Increasing numbers of contracts required analysts to work on weekends through a process that necessitated an analysis team performing tasks for clients that were allocated to other teams during the working week (thereby crossing a time boundary)

2. The client base was expanding into new international territories in which analyst teams local to the client (deemed licensees) would perform tasks on behalf of the business (crossing geographical, temporal and cultural boundaries with the business' analysts and Contract Managers).

Within these scenarios, there may not be a great requirement for the different teams to interact. However, the teams allocated to large multinational clients would be required to understand the global context of the client and would therefore need higher levels of interaction with their colleagues around the world. Within this environment, the operation of the teams aligns well with the notion of a continuum of virtualness [4]. In determining this requirement, the business recognized the importance of implementing an effective communication system within their software tools to prepare for future growth in new markets.

\section{A. Team Management}

Overcoming the communication issues that existed between the analyst teams would address a significant problem that existed within the business- working environment. However, an additional challenge lay in the management of the teams by the Contract Managers. The implementation of effective software tools to support teams has been identified as a critical success factor in team management [5], alongside training of team members and standardization of team working processes. The business had previously implemented tools to facilitate workflows for the Quality Assurance analysis that the teams would perform for clients. However, as has been discussed earlier, the growth of the business resulted in the existing tools and workflows becoming unfit for practice. Indeed, workflows had become unwieldy to the extent that analysts would work around processes rather than following defined procedures.

One of the outcomes that can result from workers not being able to work within business workflows is that the team members can develop a level of mistrust for their coworkers, leading to teams that cannot support or critique each other [6]. Clearly this would represent a significant risk to the active participation of workers within their team, or between the teams in the business. For the team leaders, the Contract Managers in the case of this business, then the statistics that could be derived from the data collated throughout a working day were at risk of being highly inaccurate, as there no longer existed a certainty that the appropriate actions had been undertaken by the analysts to meet their targets.

Equally, the ongoing professional development of the teams also became a risk. The Contract Managers were unable to determine whether training needs existed, as inaccurate statistics would mask issues that may occur. The importance of understanding the role and contribution of one worker towards the successful operation of an entire cannot be underestimated [7]. At a more holistic level for the business, then this would pose a risk to the evolution of the business, as the broader impact of a team's contribution towards the outcomes of the business could not be judged in comparison to other teams [7].

To facilitate the management of the teams in the business, both the assets and mechanisms of management would need to be considered [8]. In this context, factors may be considered to be either virtual or tangible. For the business then virtual management might be implemented through setting team targets using the software system 
whereas tangible management may be through facilitating training to overcome team issues. A virtual asset may be identified as the intrinsic client knowledge that exists within a team, whereas a tangible asset may be considered to be the collated data and reports from client sites. To ensure that management techniques could be fully supported, the company adopted the taxonomy developed by [8] to capture the styles and assets requiring support in the new software system (Table 1).

Table 1: Asset Management Taxonomy

\begin{tabular}{|l|l|l|}
\hline \multicolumn{2}{|l|}{ Assets } \\
\hline Management & Virtual & Tangible \\
\hline Virtual & $\begin{array}{l}\text { Use of integrated } \\
\text { online training } \\
\text { tools by Contract } \\
\text { Managers and } \\
\text { analysts for } \\
\text { continual personal } \\
\text { development } \\
\text { within the } \\
\text { collaboration } \\
\text { using the system } \\
\text { to collate data and } \\
\text { generate effective } \\
\text { reports for senior } \\
\text { management and } \\
\text { clients }\end{array}$ \\
\hline $\begin{array}{l}\text { Team training } \\
\text { sessions to } \\
\text { understand } \\
\text { regulatory } \\
\text { updates or } \\
\text { changing client } \\
\text { requirements. } \\
\text { Outcome of } \\
\text { training will be } \\
\text { implemented } \\
\text { through daily } \\
\text { practices that fit } \\
\text { requirements }\end{array}$ & $\begin{array}{l}\text { Team meetings to } \\
\text { identify best } \\
\text { practice and } \\
\text { training to } \\
\text { implement } \\
\text { quality assurance } \\
\text { workflows, } \\
\text { resulting in } \\
\text { published } \\
\text { workflows that } \\
\text { are fit for practice }\end{array}$ \\
\hline
\end{tabular}

\section{REFACTORING BUSINESS PROCESSES}

The refactoring project bore a high level of importance for the business in light of the continuing growth that was being experienced. Traditional approaches to the appraisal and re-engineering of workflows, such as Design Structure Matrix (DSM) [9][10] were evaluated. However, there existed two factors that were determined to bear a negative impact on the adoption of such techniques; the strong sequential interdependence between the tasks within the workflow, and the short timescales in which the refactoring process needed to be completed. Allied to this, the organisational structure of the business negated the benefits of adopting a traditional workflow analysis approach, as the business' clients required a level of independence between teams to protect commercial sensitivity (Figure 2).
In order to achieve the most appropriate and effective solution to address the cumbersome nature of the existing processes, a decision was made within the company to reengineer the quality management procedures. As the bespoke reporting toolkit used within the company had been in existence for nearly 20 years, there was a clear need to not only determine the required changes within processes to meet evolving company needs but also to drive these changes through the systems to ensure that analysts would follow the re-engineered workflows [11]. Indeed, the alignment be-tween re-engineering the technical and organizational processes was critical in gaining a successful outcome for the project [12]. There was also an identified need to integrate the company training procedures into the workflows, facilitating the adoption of past cases as training exercises for new analysts. The use of knowledge management as a means of transferring knowledge within an organization has been recognized as an effective means of cognitive collaboration [13].

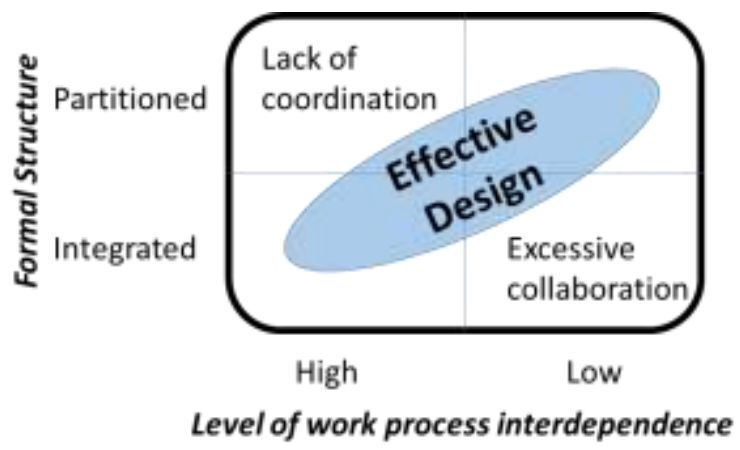

Figure 2: Relationship between work structure and process interdependence [Worren, 2012]

To better understand the impact that the user interface design process would have upon the business processes, and also to determine the potential improvements that could be achieved, the refactoring project adopted an Agile approach to the project. The Agile methodology is a software development methodology that was developed in 2001 [14] and has risen to become popular in the design and implementation of both intranet and Internet applications. A particular strength of this methodology is that it facilitates the decomposition of a system design and development to support an incremental approach. This leads to an approach that is inherently flexible as component design and implementation can be evaluated at regular short time periods, and is also evolutionary as system design can be adapted should the business requirements change during the period of the project.

To ensure that the workflow processes could be analysed and re-engineered effectively, a process of decomposition was undertaken. This process considered each task 
contained within the workflows and evaluated the steps necessary to successfully complete the process.

However, the re-engineering of the workflow process faced an additional complexity in that the existing bespoke software system did not incorporate any element of the requisite training functionality that would ensure analysts maintained and updated their skill sets in accordance with the rapidly changing operating environment of the business. Therefore there existed challenges to both realign the technical and organizational approaches [12] and to integrate training features to facilitate cognitive collaboration [13] as critical developments to ensure success of the project.

Initial investigations met with a level of resistance from the established members of the analysis teams. This led to a need for a flexible and evolutionary approach such that the user base would experience the benefits of re-engineering in an incremental manner [11]. The most popularly deployed methodology for web-based projects is the Agile methodology, or an adaptation of the Agile methodology. The agile method refers to an iterative approach that should respond to changes in requirements and inputs from clients where appropriate. The term Agile was coined in 2001 when the "Agile Manifesto" was formed [14].

An Agile based methodology was deemed most effective for this project as it allows for modules to be created and tested until stable versions are achieved. By using this methodology, incomplete sections are prevented from holding up other sections of the build, as they can be revisited and refined. The Agile methodology fits in perfectly with the chosen build method, which is modular, and MVC based. Some articles discuss the issue that when clients are involved they can often feel that this approach produces incomplete or unsatisfactory work, but where a direct client is not involved, it is a more iterative process and allows for better development, justifying its use for this project [14].

\section{IMPLEMENTATION STRATEGY}

To fulfill the potential of the Agile approach that had been adopted, the implementation of the software was designed to use the Model-View-Controller (MVC) pattern. The rationale for this decision was that the MVC model would best support an iterative design process [15]. This decision aligned with the requirement to align the process remodeling with asset remodeling to embody knowledge management [1][8]. Clearly, however, the need to address the central driver of the project (the need for improved team working and co-ordination) led to a focus on the reporting framework as a core process [16]. The reports that were constructed by analysts and Contract Managers directly informed the core business workflows

1. Quality Management for clients

2. Quality Assurance and Compliance for the business

3. Training and knowledge management for the business

\section{EVALUATION}

The refactoring project was a fifteen-month process. To achieve a successful and effective outcome this required consultation with employees and clients from all aspects of the business functions. During the course of the project, the team undertaking the task encountered resistance from some established areas of the business, such as those in IT Support, as there existed a view that the redesigned system was not an aid to assist productivity and team working, but rather a tool to micro-manage and result in unemployment amongst the existing teams in the business. The Agile approach assisted with addressing the concerns of the end users. The incremental approach allowed users to input into the process and perceive the benefits that could be achieve [11]. Equally, the iterative nature of the project allowed unforeseen enhancements to be integrated into the software system. These included the unification of a number of parallel software projects to assist analysts in completing their daily tasks, such as predictive analytics and teamwork analysis. The result of the approach has not only seen the productivity of the individual analysts, but also a determination by the teams to improve their overall productivity.

A major element of achieving the improvements in working practices has been delivered through the redesign of the user interface. The original interface did not support business workflows and required users to manipulate data from a number of different screens within the software system (or even across multiple software systems) to access and process the data required for workflow completion. The re-engineering of the user interface has offered the requisite data "at-a-glance"

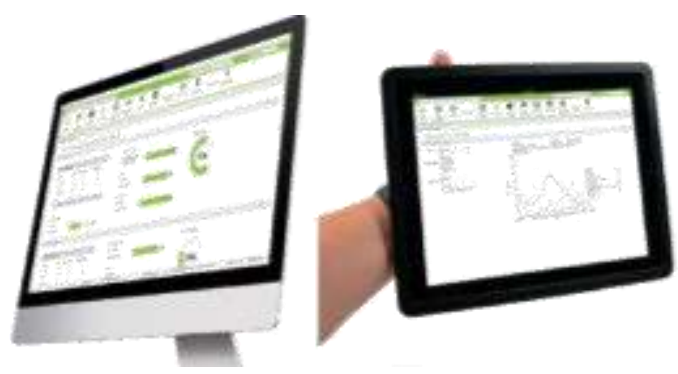

Figure 3: At a glance data presentation 
A key indicator of the success of the project, and the approach taken, has been the improved productivity of the analyst teams. This has been achieved through the time savings that have been realized through the interface redesign. Whilst the system functionality has broadly remained unchanged, the analysts have become empowered to complete tasks in a far shorter time frame than previously. Using the example adopted earlier, the fivestage process that represented seventeen hours of labor per analyst per week has now been reduced to less than one minute per week. Equally the reporting that is required of Contract Managers has been significantly improved and new functionality to facilitate the real time review of teamwork analysis has assisted in the presentation of team statistics and also the early identification of training needs within teams. Indeed, this project has also led to the integration of training tools and materials within the re-engineered system allowing analysts to take charge of their own training and provide opportunity for individuals to manage their own career progression within the business.

\section{CONCLUSION}

Any business that operates within the service sector faces numerous challenges to not only operate successfully within the trading environment, but to maintain a reputation as a leading organization within their field. This requirement becomes more critical where the environment is closely regulated, the products are high value and the service users need to maintain exacting standards in quality management. The business that has been the focus of this case study is a Small to Medium Enterprise (SME) in the United Kingdom offering quality management services. The business therefore faces extreme challenges to meet, or exceed, client requirements. When aligned with the business' reputation in its operating field, coupled with its continuing growth, then the performance of the analytic teams within the business became paramount to its success.

During a routine evaluation of the workflow practices adopted, and opportunity arose to progress the business and position its operations to support future expansion. The growth of the business meant that its employees and end users could be situated around the world, operating under different time zones and also under a range of regulatory requirements. It therefore became key to facilitate the operation of the teams such that the analysts and Contract Managers that constituted the teams would be able to interact and collaborate with colleagues around the world. This required a significant upgrade to the software systems deployed by the business to facilitate the emerging requirements of new clients.
To achieve a successful outcome to an essential refactoring project, the team employed an agile software methodology. The rationale to utilize a methodology such as this drew upon its central features of being dynamic, incremental and flexible. The strength of the approach in the context of this project aligned with changing regulatory and client demands, and end users who were resistant to change. Throughout the course of the project, all stakeholders were engaged with the development cycles, providing feedback and input to the subsequent development phases. The resultant software product is not only fit for purpose in supporting analysts and managers to complete the essential tasks for their roles, but has also provided additional benefits that were unforeseen at the start of the project. The first is that the analyst teams, and the Contract Managers, have developed a sense of urgency to improve their team performance further and enhance the environment in which they work. The second has been that the business clients have observed the analysts undertaking quality assurance work on their behalf and have extended their contracts or increased the service level that they have purchased from the business. This has led to a step change in the review process that the business applies to each of its workflows and has led to a new framework for best practice within the business.

\section{ACKNOWLEDGMENT}

Our thanks to Innovate UK and Astley Associates for their assistance and support in undertaking this project.

\section{REFERENCES}

[1] I. Kang, Y. Park and Y. Ki, "A framework for design-ing a workflow based knowledge map", Business Process Management Journal, vol. 9(3), pp 281-294, 2003

[2] Llewellyn, N. and Armistead, C. (2000), "Business process management: exploring social capital within processes", International Journal of Service Industry Management, Vol. 11 No. 3, pp. 225-43, 2000

[3] Huczynski, A.A. \& Buchanan, D.A. (2007). Organisational Behaviour (6th edition). Harlow, England: Prentice-Hall.

[4] Shin, Y. (2005). Conflict resolution in virtual teams. Organisational Dynamics, 34(4), 331-345.

[5] Duart, D.L. and Snyder, N.T. (2001). Mastering Virtual Teams: strategies, tools and techniques that succeed (2nd edition). San Francisco, CA: Jossey-Bass

[6] Ali, A., \& Kohun, F. (2007). 'Dealing with some isolation to minimize doctoral attrition - A four stage framework'. International Journal of Doctoral Studies, 2, 33-49. Available online http://www.ijds.org/Volume2/IJDSv2p033-049Ali28.pdf [Accessed 05/09/2014].

[7] Tajfel, H. \& Turner, J.C. (1986). The social identity theory of intergroup behaviour. In S. Worchel and W.G. Austin (eds), Psychology of inter-group relations (2nd edition, pp7-24). Chicago: Nelson-Hall.

[8] Warner, M. \& Witzel, M. (2004). Managing in virtual organisations. London: International Thomson Business Press.

[9] N. Worren, Organisation Design: Re-defining complex systems, Pearson, Harlow: England, 2012.

[10] S.D. Eppinger, "Innovation at the speed of information", Harvard Business Review, January, pp.149-158, 2001. 
[11] P.S. Chan and C.Land, "Implementing reengineering using information technology”, Business Process Management Journal, vol. 5(4), pp. 311-324, 1999.

[12] M.W. Salisbury, "Putting theory into practice to build knowledge management systems", Journal of Knowledge Management, vol. 7(2), pp. 128-141, 2003

[13] J. Plass and M. Salisbury, "A living system approach to the development of knowledge management systems", Educational Technology Research and Development, vol. 50(1), pp. 35-57, 2002

[14] The Agile Alliance. (2011, February 13). History: The Agile Manifesto. Retrieved October 2014 from Manifesto for Agile Software Development: http://agilemanifesto.org/history.html
[15] Haughey, D. (2010, December 23). Waterfall v Agile: How Should I Approach My Software Development Project? (Unilever) Retrieved July 31, 2014 from Project Smart: http://www.projectsmart.co.uk/waterfall-v-agile-how-should-iapproach-my-software-development-project.html

[16] M.A. Balzarova, C.J. Bamber, S. McCambridge and J.M Sharp, "Key success factors in implementation of process based management: a UK housing association experience", Business Process Management Journal, vol. 10(4), pp. 387-399, 2004 